\title{
Obesity causes cardiovascular diseases: adding to the weight of evidence
}

\author{
Aroon D Hingorani $i^{1,2,4}$, Chris Finan ${ }^{1,2,4}$, A Floriaan Schmidt ${ }^{1,3,4}$ \\ ${ }^{1}$ UCL Institute of Cardiovascular Science, London UK \\ ${ }^{2}$ Health Data Research UK, London \\ ${ }^{3}$ University Medical Centre, Utrecht, Netherlands \\ ${ }^{4}$ British Heart Foundation UCL Research Accelerator
}

The prevalence of overweight (body mass index; $\mathrm{BMI}>25 \mathrm{~kg} / \mathrm{m}^{2}$ ) and obesity $\left(\mathrm{BMI}>30 \mathrm{~kg} / \mathrm{m}^{2}\right.$ ) has been increasing in the past five decades ${ }^{12}{ }^{2}$, driven by societal changes that bring with them lifestyles characterised by lower energy expenditure and greater access to energy rich food sources than is compatible with human evolutionary adaptation.

The potential health consequences of this epidemic include increased risk of osteoarthritis of the lower limb joints ${ }^{3}$, type 2 diabetes, stroke and coronary heart disease ${ }^{4}$ and many types of cancers ${ }^{5}$. However, much of the evidence on these links comes from observational studies, a research design that can suffer limitations when attempting to infer cause-effect relationships.

Clinical trials to confirm a cause-effect relationship (and identify useful interventions for weight reduction) have proved difficult. Trials of diet and lifestyle interventions are complex to deliver and dependent on the adherence of the participants. Such trials have shown short term benefits for weight reduction, which can be difficult to sustain ${ }^{6}$, and demonstrating the effect of such interventions on clinical outcomes has also not been easy. At the other extreme, trials of different types of bariatric surgery have proven this is an effective intervention for extreme obesity leading to weight $\operatorname{loss}^{7}$ and improvement in certain health outcomes including, in some cases, reversal of type 2 diabetes ${ }^{8}$. However bariatric surgery is an expensive, resource limited intervention that is not applicable to many millions of people worldwide affected by less extreme overweight and obesity who may still be at risk of adverse health consequences and who contribute the major burden to healthcare systems. Medications developed for weight loss have had a chequered past with most proving only marginally effective and many causing serious toxicity leading to their eventual withdrawal ${ }^{9}$.

Though environmental factors have had the major role in driving the obesity epidemic it has also become clear in the last decade that body mass index and other adiposity measures are heritable traits, influenced by a large number of small effect genetic variants distributed throughout the genome ${ }^{10}$. Though their effect is small when compared to that of the environment, their discovery has opened up a new approach to investigating causal links between overweight and obesity and adverse health outcomes using Mendelian randomisation.

Mendelian randomisation is research design that will be increasingly familiar to readers of the European Heart Journal who will recognise it as a versatile tool for investigating causal relationships between risk factors and health outcomes within an observational research framework ${ }^{11}$. The principle is that if a risk factor (e.g. adiposity) is causal for a health outcome (e.g. coronary heart disease) then individuals who carry genetic variants that increase adiposity should have a higher risk of $\mathrm{CHD}$ and the effect on coronary risk should be in proportion to the effect on adiposity. The genetic associations are protected from reverse causation (since presence of the disease cannot alter the sequence of the germline) and from many types of confounding because, like treatment allocation in a clinical trial, genetic variants are allocated at random at conception according to Mendel's Laws 
(Take home Figure). Prior Mendelian randomisation analyses have provided evidence for a causal link between increased $\mathrm{BMI}$ and $\mathrm{CHD}$ and stroke $^{12}$, as well as cancer ${ }^{13}{ }^{14}$.

Against this background, Larsson and colleagues have conducted an important new analysis of over 350,000 participants from UK Biobank that sheds further light on the links between adiposity and cardiovascular disease. The study was enabled by the prospective cohort design of the UK Biobank and linkage of the participants' research data (including genotype) with routinely collected health outcomes obtained through the UK's National Health Service, which provides universal cradle tograve healthcare coverage, free at the point of delivery. Their study extends prior investigations in this arena in two ways. Firstly, it explores the effect of BMI on a wider range of cardiovascular endpoints than studied previously. Second, it also explores the influence of fat mass and fat-free mass on the same end-points using new genetic instruments identified by the authors using UK Biobank itself.

After accounting for multiple testing, the authors find evidence that a genetically-instrumented $1 \mathrm{~kg} / \mathrm{m}^{2}$ higher BMI is associated with an increased risk of eight of the fourteen cardiovascular conditions studied including: aortic stenosis, heart failure, deep venous thrombosis, hypertension, peripheral artery disease, coronary artery disease, atrial fibrillation, pulmonary embolism and subarachnoid haemorrhage (estimates in the range 6-13\% higher risk). The findings for fat mass were broadly consistent. Conversely, fat-free mass was found to exhibit inverse associations with atrial fibrillation, ischaemic stroke, as well as thoracic and aortic aneurysm. Most of these findings were consistent with those from observational epidemiology where comparisons could be drawn. However, the findings apparently diverged from observational studies for ischaemic stroke, for which the estimate was null in this analysis, though the power to detect a real effect for this endpoint was likely to have been low, and the $95 \%$ confidence limit included the potential for an important increase in risk. Mendelian randomisation studies with more stroke end-points will no doubt clarify the uncertainty here.

Although Mendelian randomisation analyses can generate critical insights on causation, a major consideration in any such study is evaluating the extent to which the inferences drawn might be compromised if the assumptions of the approach cannot be upheld. A critical assumption of this Mendelian randomisation analysis is that the genetic variants employed to instrument adiposity influence disease outcome only through the risk factor of interest ${ }^{11}$. If one or more such variants also influence other biological pathways proximal to their effect on adiposity, and some of those pathways also influence risk of the disease end points studied independent of the effects on adiposity, then the causal effect of adiposity would be overestimated due to 'horizontal pleiotropy' (Take home figure). To mitigate this, the authors undertake a number of additional analyses using recently developed methods (e.g. weighted median and Egger MR) to investigate the presence of, and account for, horizontal pleiotropy ${ }^{15,16}$. In these analyses, the effect estimates were consistent with the primary analysis using an inverse-variance weighted meta-analysis under a random effects model albeit with reduced precision.

Potential mediators of the observed effects were not explored directly in this study but the authors refer to possible candidates such as high blood pressure, glucose, low-density lipoprotein cholesterol and inflammatory cytokines such as interleukin-6, some of many of which are already targeted directly by drugs both in obese and non-obese individuals. However, prior work has suggested targeting such risk factors only attenuates about half the excess risk of coronary artery disease from an elevated $\mathrm{BMI}^{17}$. Since genomic findings can also provide new leads for drug targets ${ }^{18}$, new possibilities may emerge for safe preventative interventions can be developed that control 
established cardiovascular risk factors through the achievement and maintenance of a healthy weight and fat mass.

The findings of the current study by Larsson and colleagues lends further weight to the proposal that increased adiposity is an important causal risk factor for a wide range of cardiovascular end-points and that doctors and patients can be reasonably assured that achieving or maintaining an optimal body mass index is likely to be accompanied by widespread health benefits. The means by which this goal is to be achieved is likely to be multi-factorial including addressing the primordial societal causes that are driving weight gain, such as measures to control the advertising, availability and accessibility of calorie rich food sources on the one hand, and creating environments and infrastructures that enable greater energy expenditure as part of daily living on the other ${ }^{19}$.

\section{Figure legend}

Design and assumptions of the study by Larsson and colleagues to investigate potential causal associations of body mass index, as well as fat- and fat-free mass index with a range of cardiovascular end-points using Mendelian randomisation. (The approach, its strengths and limitations are described in more detail in the text).

1. Blüher, M. Obesity: global epidemiology and pathogenesis. Nat. Rev. Endocrinol. 15, 288-298 (2019).

2. Abarca-Gómez, L. et al. Worldwide trends in body-mass index, underweight, overweight, and obesity from 1975 to 2016: a pooled analysis of 2416 population-based measurement studies in 128.9 million children, adolescents, and adults. Lancet (2017). doi:10.1016/S01406736(17)32129-3

3. Jiang, L. et al. Body mass index and susceptibility to knee osteoarthritis: A systematic review and meta-analysis. Jt. Bone Spine (2012). doi:10.1016/j.jbspin.2011.05.015

4. MacMahon, S. et al. Body-mass index and cause-specific mortality in 900000 adults: Collaborative analyses of 57 prospective studies. Lancet (2009). doi:10.1016/S01406736(09)60318-4

5. Bhaskaran, K. et al. Body-mass index and risk of 22 specific cancers: A population-based cohort study of 5·24 million UK adults. Lancet (2014). doi:10.1016/S0140-6736(14)60892-8

6. Franz, M. J. et al. \{A figure is presented\}Weight-Loss Outcomes: A Systematic Review and Meta-Analysis of Weight-Loss Clinical Trials with a Minimum 1-Year Follow-Up. J. Am. Diet. Assoc. (2007). doi:10.1016/j.jada.2007.07.017

7. Colquitt, J. L., Pickett, K., Loveman, E. \& Frampton, G. K. Surgery for weight loss in adults. Cochrane Database of Systematic Reviews (2014). doi:10.1002/14651858.CD003641.pub4

8. Gloy, V. L. et al. Bariatric surgery versus non-surgical treatment for obesity: a systematic review and meta-analysis of randomised controlled trials. BMJ (2013). doi:10.1136/bmj.f5934

9. Cheung, B. M. Y., Cheung, T. T. \& Samaranayake, N. R. Safety of antiobesity drugs. Ther. Adv. Drug Saf. (2013). doi:10.1177/2042098613489721

10. Locke, A. E. et al. Genetic studies of body mass index yield new insights for obesity biology. Nature 518, (2015). 
11. Smith, G. D. \& Hemani, G. Mendelian randomization: Geneticanchorsfor causal inference in epidemiological studies. Hum. Mol. Genet. 23, 1-10 (2014).

12. Dale, C. E. et al. Causal Associations of Adiposity and Body Fat Distribution with Coronary Heart Disease, Stroke Subtypes, and Type 2 Diabetes Mellitus: A Mendelian Randomization Analysis. Circulation 135, (2017).

13. Thrift, A. P. et al. Obesity and risk of esophageal adenocarcinoma and barrett's esophagus: A mendelian randomization study. J. Natl. Cancer Inst. (2014). doi:10.1093/jnci/dju252

14. Thrift, A. P. et al. Mendelian randomization study of body mass index and colorectal cancer risk. Cancer Epidemiol. Biomarkers Prev. (2015). doi:10.1158/1055-9965.EPI-14-1309

15. Bowden, J., Smith, G. D. \& Burgess, S. Mendelian randomization with invalid instruments: Effect estimation and bias detection through Egger regression. Int. J. Epidemiol. (2015). doi:10.1093/ije/dyv080

16. Bowden, J., Davey Smith, G., Haycock, P. C. \& Burgess, S. Consistent Estimation in Mendelian Randomization with Some Invalid Instruments Using a Weighted Median Estimator. Genet. Epidemiol. (2016). doi:10.1002/gepi.21965

17. Danaei, G. Metabolic mediators of the effects of body-mass index, overweight, and obesity on coronary heart disease and stroke: A pooled analysis of 97 prospective cohorts with 1.8 million participants. Lancet (2014). doi:10.1016/S0140-6736(13)61836-X

18. Finan, C. et al. The druggable genome and support for target identification and validation in drug development. Sci. Transl. Med. 9, (2017).

19. WHO Europe. http://www.euro.who.int/en/health-topics/noncommunicablediseases/obesity/policy (2019). 